\title{
SURFACES WITH MAXIMAL LIPSCHITZ-KILLING CURVATURE IN THE DIRECTION OF MEAN CURVATURE VECTOR
}

\author{
CHORNG-SHI HOUH
}

\begin{abstract}
M^{2}$ is an oriented surface in $E^{2+N}$. If $M^{2}$ is pseudoumbilical, the Lipschitz-Killing curvature takes maximum in the direction of mean curvature vector. The converse is also investigated. Furthermore assuming that $M^{2}$ is closed, pseudo-umbilical and its Gaussian curvature has some nonnegative lower bound, $M^{2}$ is completely determined by the $M$-index of $M^{2}$.
\end{abstract}

1. Let $M^{2}$ be an oriented Riemannian surface with an isometric immersion $x: M^{2} \rightarrow E^{2+N}$ in a euclidean space $E^{2+N}$. Let $F\left(M^{2}\right)$ and $F\left(E^{2+N}\right)$ be the bundles of orthonormal frames of $M^{2}$ and $E^{2+N}$ respectively. Throughout this paper we assume that the mean curvature vector $H$ of $M^{2}$ is nowhere zero. Let $B$ be the set of elements $b=\left(p, e_{1}, e_{2}, \cdots, e_{2+N}\right)$ such that $\left(p, e_{1}, e_{2}\right) \in F\left(M^{2}\right), e_{3}=H /|H|$ and that $\left(x(p), e_{1}, e_{2}, e_{3}, \cdots, e_{2+N}\right) \in$ $F\left(E^{2+N}\right)$ whose orientation is coherent with that of $E^{2+N}$, identifying $e_{i}$ with $d x\left(e_{i}\right), i=1,2$. Let $\tilde{x}: B \rightarrow F\left(E^{2+N}\right)$ be the mapping naturally defined by $\tilde{x}(b)=\left(x(p), e_{1}, \cdots, e_{2+N}\right)$.

We have the differential forms $\omega_{i}, \omega_{i j}, \omega_{i \alpha}, \omega_{\alpha \beta}(1 \leqq i, j \leqq 2,3 \leqq \alpha$, $\beta \leqq 2+N)$ on $B$ derived from the basic forms and the connection forms on $F\left(E^{2+N}\right)$ through $\tilde{x}$ as follows.

$$
\begin{aligned}
& d x=\omega_{1} e_{1}+\omega_{2} e_{2}, \quad d e_{A}=\sum_{B=1}^{2+N} \omega_{A B} e_{B}, \quad \omega_{A B}=-\omega_{B A} \\
& (A, B=1,2, \cdots, 2+N) ; \\
& \omega_{i \alpha}=\sum_{j=1}^{2} A_{\alpha i j} \omega_{j}, \quad A_{\alpha i j}=A_{\alpha j i} .
\end{aligned}
$$

In the following, for the summation notations $\Sigma_{l}, \Sigma_{\alpha}$ and $\Sigma_{r}$ we mean $\sum_{i=1}^{2}, \sum_{\alpha=3}^{2+N}$ and $\sum_{r=4}^{2+N}$, for the indices $i, j, \alpha, \beta, r, t$ we mean $1 \leqq i, j \leqq 2$, $3 \leqq \alpha, \beta \leqq 2+N, 4 \leqq r, t \leqq 2+N$.

Received by the editors February 4, 1972.

AMS 1970 subject classifications. Primary 53C45; Secondary 53A05,

Key words and phrases. Lipschitz-Killing curvature, mean curvature vector, $M$ index, normal curvature, pseudo-umbilical, scalar normal curvature.

(c American Mathematical Society 1972 
Now we choose $e_{1}, e_{2}$ as the principal directions of $e_{3}$, then with respect to the frame $\left(e_{1}, e_{2}, e_{3}, \cdots, e_{2+N}\right)$ the matrices $A_{\alpha}=\left(A_{\alpha i j}\right)$ are written in

$$
\left(A_{3 i j}\right)=\left(\begin{array}{ll}
a & 0 \\
0 & b
\end{array}\right), \quad\left(A_{r i j}\right)=\left(\begin{array}{cc}
c_{r} & d_{r} \\
d_{r} & -c_{r}
\end{array}\right) .
$$

$H=\frac{1}{2}(a+b) e_{3}$. When $a=b, M^{2}$ is pseudo-umbilical. Let $e$ be a unit normal vector to the tangent plane $d x\left(T_{p}\left(M^{2}\right)\right)$ at $x(p)$. Then

$$
e=\sum_{\alpha} \xi_{\alpha} e_{\alpha}, \quad \sum_{\alpha} \xi_{\alpha}^{2}=1
$$

Let $A(e)$ be the following matrix

$$
A(e)=\sum_{\alpha} \xi_{\alpha} A_{\alpha}=\left(\begin{array}{cc}
a \xi_{3}+\sum_{r} c_{r} \xi_{r} & \sum_{r} d_{r} \xi_{r} \\
\sum_{r} d_{r} \xi_{r} & b \xi_{3}-\sum_{r} c_{r} \xi_{r}
\end{array}\right) .
$$

The Lipschitz-Killing curvature $G(p, e)$ is given by [4]

$$
\begin{aligned}
G(p, e) & =\operatorname{det}(A(e)) \\
& =a b \xi_{3}^{2}+(b-a) \xi_{3} \sum_{r} c_{r} \xi_{r}-\left(\sum_{r} c_{r} \xi_{r}\right)^{2}-\left(\sum_{r} d_{r} \xi_{r}\right)^{2} .
\end{aligned}
$$

Let $S_{2}$ be the set of all real symmetric square matrices of order 2 . Let $m: S_{2} \rightarrow R$ be a linear transformation defined by [6] $m(A)=\frac{1}{2}$ trace $A$, $A \in S_{2}$. We denote the normal space to $x\left(M^{2}\right)$ at $x(p)$ by $N_{p}, N_{p}=$ $\left\{X, X=\sum_{\alpha} \eta_{x} e_{\alpha}, \eta_{x} \in R\right\}$, and define a linear mapping $\bar{m}: N_{p} \rightarrow R$ by

$$
\bar{m}(X)=\sum_{\alpha} \eta_{\alpha} m\left(A_{\alpha}\right), \quad X=\sum_{\alpha} \eta_{\alpha} e_{\alpha} .
$$

The kernel of $\bar{m}$ is denoted by ker $\bar{m}$.

At any point $p \in M^{2}$ we take a frame $b=\left(p, e_{1}, \cdots, e_{2+.}\right) \in B$. Let $\psi_{b}: N_{p} \rightarrow S_{2}$ be the linear mapping defined by

$$
\psi_{b}\left(\sum_{\alpha} \eta_{\alpha} e_{\alpha}\right)=\sum_{\alpha} \eta_{\alpha} A_{\alpha} .
$$

The dimension of $\psi_{b}(\operatorname{ker} \bar{m})$ is called the $M$-index of $M^{2}$ at $p$ and is denoted by $M$-index $M^{2}[6]$.

2. We prove the following lemma.

Lemma. If at any point $p \in M^{2}$ the Lipschitz-Killing curvature $G(p, e)$ has maximum in the direction of $H$ then $a b \geqq 0$ where $a, b$ are given by (1). 
Proof. $e_{3}=H /|H|$, which is given by $\xi_{3}=1, \xi_{r}=0$ in (2). By (2) and (3) it is easy to see that if $G(p, e)$ takes maximum at $\xi_{3}=1, \xi_{r}=0$ then $(a-b) c_{r}=0$. Hence by (3) the maximum of $G(p, e)$ is $a b$. Now let $S_{p}$ be an arbitrary chosen unit circle in $N_{p}$ and $e^{\prime}$ be a fixed point in $S_{p}$. Put $S_{p}^{*}=$ $S_{p}-\left\{e^{\prime}\right\}$. We choose $e \in S_{p}^{*}$ and $e_{1}(e), e_{2}(e)$ as two unit orthogonal tangent vectors in the principal directions of $e$ and move $e$ differentiably on $S_{p}^{*}$. Then the principal curvatures $k_{1}(e)$ and $k_{2}(e)$ with respect to $e_{1}(e)$ and $e_{2}(e)$ are continuous on $S_{p}^{*}$. Now suppose $k_{1}(e) \neq 0$ at some $e \in S_{p}^{*}$. Then by the continuity of $k_{1}$ on $S^{*}$ and the fact $k_{1}(-e)=-k_{1}(e)$ we see that $k_{1}=0$ for some points in $S_{p}^{*}$. This implies that the Lipschitz-Killing curvature $G(p, e)=0$ for some $e \in S_{p}^{*}$. Since $a b$ is the maximum of $G(p, e)$ we conclude that $a b \geqq 0$. This is true for all $p \in M^{2}$.

3. If $M^{2}$ is pseudo-umbilical then $a=b$ and by (3) we have that $G(p, e)$ takes maximum in $e_{3}$. To get further results we consider the normal curvature $R_{\beta i j}^{\alpha}$ and scalar normal curvature $K_{. V}[1]$ :

$$
\begin{aligned}
R_{\beta k l}^{\alpha} & =\sum_{i}\left(A_{\alpha i k} A_{\beta i l}-A_{\alpha i l} A_{\beta i k}\right), \\
K_{. V} & =\sum_{\alpha, \beta, i, j}\left(A_{\alpha i k} A_{\beta j k}-A_{\alpha j k} A_{\beta i k}\right)^{2} .
\end{aligned}
$$

TheOReM 1. At points $p$ with $M$-index $M^{2} \geqq 2, M^{2}$ is pseudo-umbilical if and only if $\left(j(p, e)\right.$ has maximum in $e_{3}$; at points $p$ with $M$-index $M^{2}=1$, $M^{2}$ is pseudo-umbilical if and only if $G(p, e)$ has maximum in $e_{3}$ and $K_{N}=0$; at points $p$ with $M$-inde $x_{p} M^{2}=0, M^{2}$ is pseudo-umbilical if and only if $M^{2}$ is totally umbilical.

Proof. Suppose $M$-index $M^{2} \geqq 2$. Otsuki in $[6]$ showed that $M$ index $_{p} M^{2} \leqq 2$, so $M$-index ${ }_{2} M^{2}=2$. Since $m\left(A_{3}\right)=\frac{1}{2}(a+b) \neq 0$ and $m\left(A_{r}\right)=$ 0 we have ker $\bar{m}=\left\{\sum_{r} \eta_{r} e_{r}, \eta_{r} \in R\right\}, e_{r} \in \operatorname{ker} \bar{m}$ and $\psi_{b}\left(e_{r}\right)=A_{r}$. Hence for at least one $r, c_{r} \neq 0$. But $G(p, e)$ takes maximum in $e_{3}$; we have $(a-b) c_{r}=0$. Hence $a=b$ and $M^{2}$ is pseudo-umbilical. Next suppose $M$-index $M^{2}=1$, $G(p, e)$ has maxim!m in $e_{3}$ and $K_{\Lambda}=0 . A_{r}$ are given in $(1) . \psi_{b}(\operatorname{ker} \bar{m})=$ $\left\{\sum_{r} \eta_{r} A_{r}, \eta_{i r}=R_{j}\right.$. If $\operatorname{dim}\left(\psi_{b}(\operatorname{ker} \bar{m})\right)=M$-inde. $x_{p} M^{2}=1$ then there is $k$ so that $d_{r}=k c_{r}$ for any $r$. We have then $A_{r} A_{t}=A_{t} A_{r}$ and

$$
K_{\Lambda}=2 \sum_{\beta, i, j}\left(A_{3 i k} A_{\beta j k}-A_{3 j k} A_{\beta, k}\right)^{2} .
$$

$K_{N}=0$ implies $A_{3} A_{r}=A_{r} A_{3}$, that is $(a-b) d_{r}=0$. If all $\iota_{r}=0$ then at least one $c_{r} \neq 0$ because $M$-index $M^{2}=1$. Thus $G(p, e)$ having maximum at $e_{3}$ implies $a=b$. The inverse is clear. Finally suppose $M$-index $M^{2}=0$, then $c_{r}=d_{r}=0$, i.t., $A_{r}=0$. It is clear that $M^{2}$ is pseudo-umbilical. i.e., $a=b$, if and only if $M^{2}$ is totally umbilical. 
4. In this section we assume that $M^{2}$ is a closed surface. For a symmetric matrix $A=\left(a_{i j}\right)$ if we write $N(A)=\sum_{i, j} a_{i j}^{2}$, then we have $K_{N}=$ $\sum_{\alpha, \beta} N\left(A_{\alpha} A_{\beta}-A_{\beta} A_{\alpha}\right)$. Chen in [1] proved the following results: The Veronese surface is the only closed pseudo-umbilical surface in euclidean space with parallel normal curvature and scalar normal curvature $K_{N} \neq 0$, the 2-sphere and the Clifford torus are the only closed pseudo-umbilical surfaces in euclidean space with scalar normal curvature $K_{N}=0$ and scalar curvature $R \geqq 0$. From these results we have the following two theorems.

THEOREM 2. If $M^{2}$ is closed, pseudo-umbilical, $M$-index $M_{p}^{2}=1$ for any $p$ and the Gaussian curvature $G(p) \geqq 0$ everywhere then $M^{2}$ is either a sphere or a Clifford torus.

Proof. If $M^{2}$ is pseudo-umbilical and $M$-index ${ }_{p} M^{2}=1$ we have by Theorem 1 that $K_{N}=0$. The scalar curvature $R=2 G \geqq 0$ by assumption. By Chen's result $M^{2}$ is either a sphere or a Clifford torus.

THEOREM 3. If $M^{2}$ is closed, pseudo-umbilical, $M$-index ${ }_{p} M^{2}=2$ for any $p$ and the Gaussian curvature $G(p) \geqq(N-2) a^{2} / 2 N-3$ everywhere then $M^{2}$ is a Veronese surface.

Proof. $M$-index ${ }_{p} M^{2}=2$ implies that $K_{N} \neq 0$. For the Laplacian of $A_{\alpha i j}$ in the case of pseudo-umbilical $M^{2}$ we have the known equality [2]:

$$
\sum_{\alpha, i, j} A_{\alpha i j} \Delta A_{\alpha \imath j}=2 a \Delta a+2 a^{2} S-\sum_{\alpha} S_{\alpha}^{2}-\sum_{\alpha \neq \beta} N\left(A_{\alpha} A_{\beta}-A_{\beta} A_{\alpha}\right)
$$

where $S_{\alpha}=\sum_{i, j} A_{\alpha i j}^{2}=N\left(A_{\alpha}\right), S=\sum_{\alpha} S_{\alpha}$. Since $M^{2}$ is pseudo-umbilical we have $A_{3}=a l$ and $\sum_{i, j} A_{3 i j} \Delta A_{3 i j}=2 a \Delta a$. It is known also that [3] $N\left(A_{\alpha} A_{\beta}-A_{\beta} A_{\alpha}\right) \leqq 2 N\left(A_{\alpha}\right) N\left(A_{\beta}\right)$. So we have $\sum_{r \neq t} N\left(A_{r} A_{t}-A_{t} A_{r}\right) \leqq$ $2 \sum_{r \neq t} N\left(A_{r}\right) N\left(A_{t}\right)=2 \sum_{r \neq t} S_{r} S_{t}$. Let $\bar{S}=\sum_{r} S_{r}$ and noticing that $2 a^{2} S_{3}=$ $2 a^{2}\left(2 a^{2}\right)=4 a^{4}=S_{3}^{2}$ we have from (7):

$$
\begin{aligned}
\sum_{r, i, j} A_{r i j} \Delta A_{r i j} & \geqq 2 a^{2} \bar{S}-\sum_{r} S_{r}^{2}-2 \sum_{r \neq t} S_{r} S_{t} \\
& =2 a^{2} \bar{S}-\left(\sum_{r} S_{r}\right)^{2}-2 \sum_{r<t} S_{r} S_{t}
\end{aligned}
$$

Let $\sigma_{1}, \quad \sigma_{2}$ be such that $(N-1) \sigma_{1}=\sum_{r} S_{r}=S,\left(\frac{1}{2}\right)(N-1)(N-2) \sigma_{2}=$ $\sum_{r<t} S_{r} S_{t}$; it can easily be seen that [3]: $(N-1)^{2}(N-2)\left(\sigma_{1}^{2}-\sigma_{2}\right)=$ $\sum_{r<t}^{r<t}\left(S_{r}-S_{t}\right)^{2} \geqq 0$. Hence $\sigma_{1}^{2} \geqq \sigma_{2}$ or

$$
2 \sum_{r<t} S_{r} S_{t} \leqq(N-2) \bar{S}^{2} /(N-\mathbf{1})
$$


By (8) and (9) we have

$$
\sum_{r, i, j} A_{r i j} \Delta A_{r i j} \geqq-(2 N-3) \bar{S}^{2} /(N-1)+2 a^{2} \bar{S}
$$

The Gaussian curvature of $M^{2}$ is $G(p)=\sum_{\alpha} \operatorname{det}\left(A_{\alpha}\right)=a^{2}-\sum_{r}\left(c_{r}^{2}+d_{r}^{2}\right)=$ $a^{2}-\left(\frac{1}{2}\right) S$. Therefore $S=2\left(a^{2}-G\right)$. By (10) we have

$$
-\sum_{r, i, j} A_{r i j} \Delta A_{r i j} \leqq 2 \bar{S}\left[(2 N-3)\left(a^{2}-G\right) /(N-1)-a^{2}\right] .
$$

Thus if $(2 N-3)\left(a^{2}-G\right) /(N-1)-a^{2} \leqq 0$ or $G \geqq(N-2) a^{2} /(2 N-3)$ then $\sum_{r, i, j} A_{r i j} \Delta A_{r i j} \geqq 0$. Now from the equality

$$
\left(\frac{1}{2}\right) \Delta \sum_{r, i, j}\left(A_{r i j}\right)^{2}=\sum_{r, i, j, k}\left(A_{r i j k}\right)^{2}+\sum_{r, i, j} A_{r i j} \Delta A_{r i j},
$$

where $A_{r i j k}=A_{r i j, k}$ (covariant derivative), we have that if $G \geqq$ $(N-2) a^{2} /(2 N-3)$ then $\Delta \sum_{r, i, j}\left(A_{r i j}\right)^{2} \geqq 0$. Since $M^{2}$ is closed we have $\Delta \sum_{r, i, j}\left(A_{r i j}\right)^{2}=0$. By (11) it implies $A_{r i j, k}=0$. By (6) we have that $R_{\beta k l}^{\alpha}$ (noticing that $R_{\beta k l}^{3} \equiv 0$ ) is parallel and that $K_{N}$ is constant and $K_{N} \neq 0$. By Chen's result we conclude that $M^{2}$ is a Veronese surface.

THEOREM 4. If $M^{2}$ is closed, $G(p, e)$ takes maximum in $e_{3}$ and $M$-index $M^{2}=0$ everywhere then $M^{2}$ is embedded as a convex surface in an $E^{3}$.

Proof. $M$-index ${ }_{p} M^{2}=0$ implies that $c_{r}=d_{r}=0$. Then we may write $G(p, e)=a b \cos ^{2} \theta_{3}, 0 \leqq \theta_{3} \leqq \pi$. By the lemma $a b \geqq 0$. The Gaussian curvature $G(p)$ in this case is $G(p)=\sum_{\alpha} \operatorname{det}\left(A_{\alpha}\right)=a b$. So we have $G(p) \geqq G(p, e)$ for all $e$. By the Gauss-Bonnet formula $\int_{M^{2}} G(p) d V=\int_{M^{2}} a b d V=4 \pi(1-g)$. On the other hand the total curvature $K^{*}(p)$ of $M^{2}$ at $p$ is

$$
K^{*}(p)=\int_{S^{N-1}}|G(p, e)| d \sigma_{N-1}=\int_{S^{N-1}} a b \cos ^{2} \theta_{3} d \sigma_{N-1}=G(p) c_{N+1} / 2 \pi
$$

where $S^{N-1}$ is the unit sphere in $E^{N}$ and $c_{N+1}$ is the volume of the unit sphere in $E^{2+N}$.

By a result due to Chern-Lashof [5] we have $\left(1 / c_{N+1}\right) \int_{M^{2}} K^{*}(p) d V \geqq$ $2+2 g$, the equality sign holds if and only if $M^{2}$ is embedded as a convex surface in an $E^{3}$. On the other hand

$$
\begin{aligned}
\left(c_{N+1} / 2 \pi\right) 4 \pi(1-g) & =\left(c_{N+1} / 2 \pi\right) \int_{I^{2}} G(p) d V \\
& =\int_{.1^{2}} K^{*}(p) d V \geqq c_{N+1}(2+2 g) .
\end{aligned}
$$

That is $1-g \geqq 1+g$. Thus it is necessary that $g=0$ and the equality sign holds. Hence $M^{2}$ is embedded as a convex surface in $E^{3}$. 
The author would like to express his thanks to Dr. B. Y. Chen for the valuable conversations.

\section{REFERENCES}

1. B. Y. Chen, Pseudo-umbilical submanifolds of a Riemannian manifold of constant curvature. III, J. Differential Geometry (to appear).

2. - Some results of Chern-Do Carmo-Kobayashi type and the length of second fundamental form, Indian Math. J. 20 (1970), 1175-1185.

3. S. S. Chern, M. Do Carmo and S. Kobayashi, Minimal submanifolds of a sphere with second fundamental form of constant length, Proc. Conf. on Functional Analysis and Related Fields (Univ. of Chicago, Chicago, Ill., 1968), Springer, New York, 1970, pp. 59-75. MR 42 \#8424.

4. S. S. Chern and R. K. Lashof, On the total curvature of immersed manifolds, Amer. J. Math. 79 (1957), 306-318. MR 18, 927.

5. - On the total curvature of immersed manifolds. II, Michigan Math. J. 5 (1958), 5-12. MR 20 \#4301.

6. T. Otsuki, $A$ theory of Riemannian submanifolds, Kōdai Math. Sem. Rep. 20 (1968), 282-295. MR 38 \#2707.

Department of Mathematics, Wayne State University, Detroit, Michigan 48202 